\title{
Resistance to extinction in classical GSR conditioning
}

\author{
H. D. KIMMEL and D. W. OWEN \\ Liniersity of South Florida. Tampa. Fla. 36620
}

Two groups of $15 \mathrm{Ss}$, matched in number of paired conditioning trials needed to attain peak GSR magnitude, were then differentiated by giving one group 16 paired postpeak trials and the other 16 unpaired CSs and UCSs. Both groups were then given CS-only extinction trials until either two zero responses or 50 trials occurred. The unpaired postpeak group extinguished in an average of 2.6 trials, while the paired postpeak group extinguished in 10.6 trials. This difference was highly statistically significant. The unpaired postpeak group's mean GSR during acquisition was below that of the paired postpeak group only on the last block of trials. Administering paired prepeak trials apparently potentiates the extinguishing effect of unpaired trials subsequently delivered, possibly via some kind of "contrast" effect, since the unpaired postpeak group extinguished faster than occurs when no pairing whatsoever is given.

When human Ss are given paired CS-UCS presentations beyond that point in the acquisition process at which anticipatory GSRs to the CS have ceased to increase in magnitude and begun to decrease (postpeak paired trials), resistance to extinction also declines (Silver \& Kimmel, 1969: Schramm \& Kimmel, 1970 ). When only two postpeak paired trials are given, Ss require approximately $40-45$ presentations of the CS alone to reach an extinction criterion of two successive zero responses. When 16 postpeak paired trials are administered, the extinction criterion is reached within about 8-14 trials on the CS alone. The latter range is almost identical to what is shown by Ss who receive completely unpaired CS and UCS presentations (matched in number to those given to the paired Ss) and then are extinguished on the CS alone.

These findings have been attributed (Schramm \& Kimmel, 1970) to a hypothetical conditioned inhibitory process, perhaps akin to Pavlovian inhibition of delay, which is assumed to result in CR attenuation following an initial excitatory phase and, eventually, rapid extinction. It remains to be established, however, whether both pre- and postpeak pairing are necessary factors in this process. The control Ss in the Silver \& Kimmel (1969) and Schramm \& Kimmel (1970) studies were given unpaired trials during "acquisition" and then were extinguished on the CS alone. These studies did not include controls run with initial paired acquisition trials, up to the peak CR, followed by postpeak unpaired trials and, then, CS-only extinction. If postpeak pairing was an essential factor in these instances of attenuated resistance to extinction, unpaired postpeak trials following initial pairing should not result in as much reduction of resistance to extinction. The present study was designed to examine this question.

\section{METHOD}

Thirty undergraduate psychology students volunteered to serve as Ss to receive extra class points. Pairs of Ss were created. by matching them in number of paired trials needed to attain the peak CR, with one group of 15 Ss then receiving 16 postpeak paired trials and the other receiving 16 postpeak unpaired CS and UCSs. Since one postpeak trial is needed to determine that the peak has occurred. a total of 17 postpeak trials (or unpaired CSs and UCSs) were administered. All Ss were then given extinction trials on the CS lnne until either two successive nonresponses or 50 trials occurred.

Equipment and general procedures were idintical to that of the Schramm \& Kimmel (1970) study. The CS was a 40-dB (physical reference) $1-\mathrm{kHz}$ pure tone. delivered via earphones. and the UCS was a $4.0-\mathrm{mA}$ shock to the volar surface of the left forearm. CS duration was $5.0 \mathrm{sec}$. LCS duration was $0.1 \mathrm{sec}$, and both terminated simultaneously. The ITI for paired trials was between 30 and $70 \mathrm{sec}$. with a mean of $50 \mathrm{sec}$, while for unpaired presentations these values were halved. The GSR was picked up from the palm and back of the right hand and was amplified and recorded at a paper speed of $4 \mathrm{in} . / \mathrm{min}$. Data were collected in an IAC audiometric chamber. The GSR response was measured within a 5 -sec interval beginning $1 \mathrm{sec}$ after $\mathrm{CS}$ onset.

\section{RESULTS AND DISCUSSION}

Of principal interest was the number of trials needed to reach the extinction criterion in the two experimental groups. The Ss who received 16 postpeak paired trials required an average of 10.6 extinction trials. a value almost exactly in the middle of the range previously found under this condition (Silver \& Kimmel. 1969: Schramm \& Kimmel, 1970). However, the Ss who received postpeak unpaired trials extinguished in an average of 2.6 trials, a result in the opposite direction from that expected. The difference between the two groups' mean numbers of extinction trials was highly significantly in favor of the paired postpeak condition, $t$ $=3.15, \mathrm{p}<.01$.

Figure 1 shows the average GSR (change in log conductance) of the paired postpeak (experimental) and unpaired postpeak (control) groups on the peak trial and during the subsequent postpeak trials (in two-trial blocks). As would be expected from the matching procedure, the two groups' average GSRs did not differ significantly on the peak trial $(t=0.01)$. Although the groups' curves were similar during postpeak trials, the paired postpeak group's mean was significantly higher than that of the unpaired postpeak group's on the last block $(\mathrm{t}=2.52, \mathrm{p}<.05)$. Otherwise. there were no significant acquisition differences between the groups. Because more than half of the Ss in the unpaired postpeak group reached the extinction criterion in the first two extinction trials (while none of the Ss in the paired postpeak group did), no statistical analysis of the extinction GSR magnitude data was done.

Besides the obvious fact that the Ss who received unpaired postpeak trials extinguished more rapidly than 


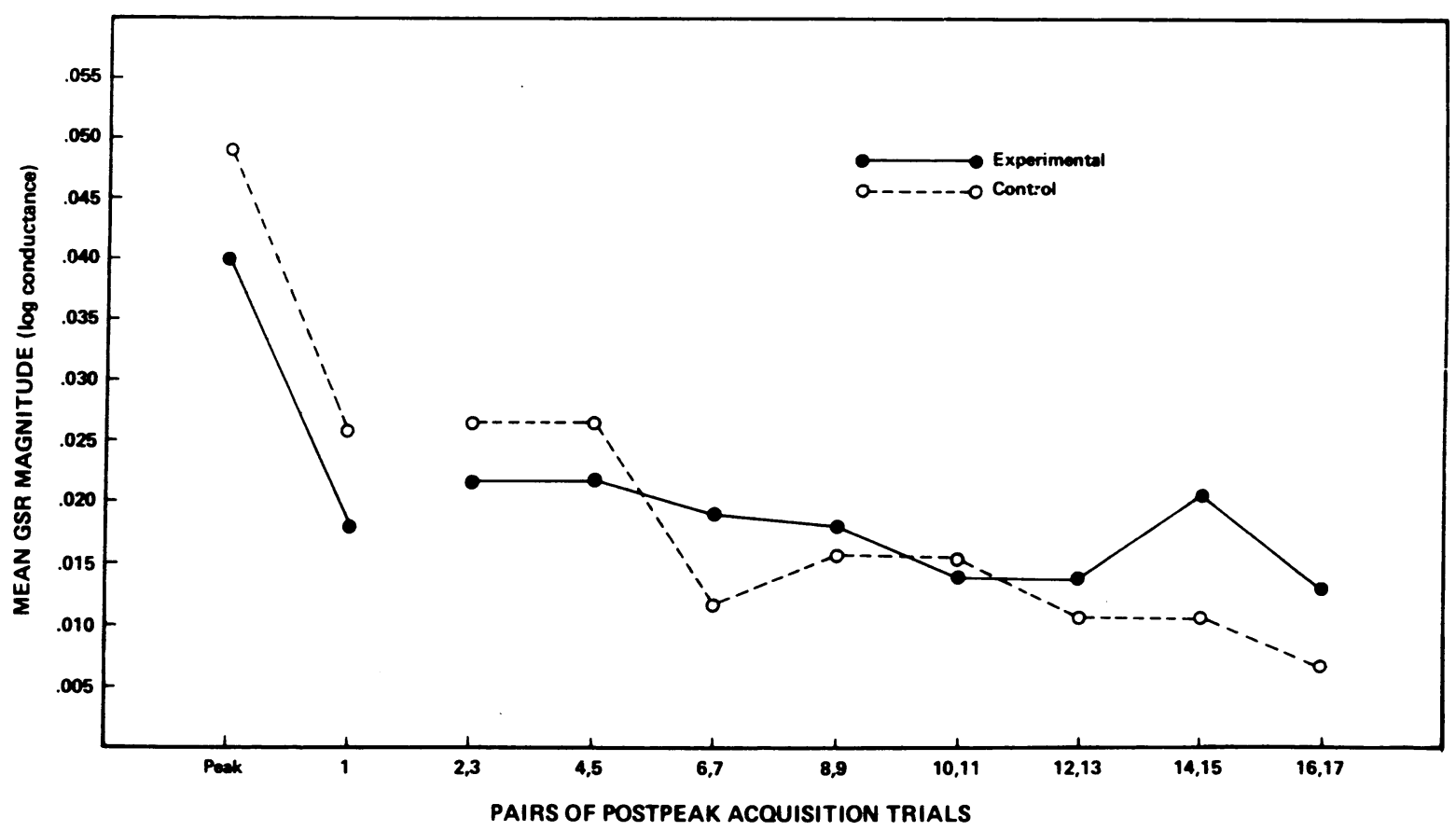

Fig. 1. Mean GSR magnitude (change in log conductance) of paired postpeak (experimental) and unpaired postpeak (control) groups on peak and subsequent acquisition trials (in two-trial blocks).

those who received paired postpeak trials, it should be noted that the unpaired postpeak group also extinguished far more rapidly than Ss in previous studies who received nothing but unpaired CSs and UCSs prior to extinction. In the Silver \& Kimmel (1969) study, the control Ss were given an average of about 20 unpaired CSs and UCSs prior to extinction, and they required 14 trials to attain the extinction criterion. A comparable control group in the Schramm \& Kimmel (1970) experiment needed 12 extinction trials to reach criterion. Apparently, Ss who are given initial paired CS-UCS trials followed by unpaired CSs and UCSs extinguish much more rapidly than Ss who are never given any paired trials. In other words, the rapid extinction of the present paired-unpaired control group cannot be attributed to "extinction" which was occurring during the postpeak unpaired sequence, before our "formal" extinction series began. Ss who are never given any paired trials (i.e., who receive nothing but unpaired CSs and UCSs) take longer to extinguish than the present Ss who were first given a few paired trials and then received unpaired CSs and UCSs. The initial series of paired trials must somehow potentiate the extinguishing effect of subsequent unpaired CSs and UCSs, perhaps via a kind of "contrast"? effect. Further evidence that the unpaired condition is not simply one in which "extinction". is taking place before "formal" extinction is begun may be seen in the finding of Schramm \& Kimmel (1970) that the number of unpaired CSs and UCSs does not influence resistance to extinction in a completely unpaired condition. They found that a completely unpaired group which received 8 unpaired
CSs and UCSs reached the extinction criterion in 13 trials (their Group U-4), while another completely unpaired group which received 20 unpaired CSs and UCSs reached criterion in 12 trials (their Group U-16). The present paired-unpaired control group (with about 5 paired trials and 16 unpaired CSs and UCSs) extinguished in 2.6 trials.

The present use of the term "contrast" is not meant to imply a cognitive process, nor is it intended to suggest that an explanation of the effect is apparent. What is meant is nothing more nor less than the fact that the experimental procedure of presenting unpaired CSs and UCSs following pairing is to accelerate the tendency for CS-only presentations (i.e., standard extinction) to produce nonresponding to the CS. This experimental procedure results in far more rapid loss of responding than that produced by an equivalent number of unpaired CSs and UCSs following initial unpaired CSs and UCSs, or, even, by an equivalent number of presentations of , the CS alone. ${ }^{1}$ The mechanism underlying this effect would appear to be a deserving subject for future study.

\section{REFERENCES}

Schramm, C. F., \& Kimmel, H. D. Resistance to extinction in GSR conditioning following different numbers of postpeak acquisition trials. Journal of Experimental Psychology, 1970, 84, 239-243.

Silver, A. I., \& Kimmel, H. D. Resistance to extinction in classical GSR conditioning as a function of acquisition trials beyond peak CR size. Psychonomic Science, 1969, 14, 54-55.

NOTE

1. Silver \& Kimmel (1969) found that Ss given standard 
CS-only extinction trials following the peak acquisition conditioned GSR extinguished in an average of 42 trials, while Schramm \& Kimmel (1970) obtained an average of 44 trials under the same conditions. If the 16 unpaired CSs and UCSs of the present paired-unpaired controls are assumed to be the approximate equiralent of 16 extinction trials, then these Ss reached the nonresponse criterion in a total of 18.6 trials (i.e.. 16 plus 2.6), still substantially less than the values obtained in the standard CS-only extinction condition.

(Received for publication November 5. 1973.)

\title{
The effect of differential overtraining of the positive and negative stimulus on the aversiveness of the negative stimulus
}

\author{
ISAAC BEHAR* \\ United States Army Medical Research Laboratory, Fort Knox, Kentucky 40121
}

\begin{abstract}
Six discrimination-sophisticated monkeys were trained on 18 problems, each of which contained intermixed trials on two discriminations. In six problems, the ratio of trials of the two discriminations was 4 to 1 . Following training, a single test trial was given, which consisted of choice between the more trained (MT) and less trained (LT) negative stimuli. No consistent preference for either stimulus was demonstrated. In the remaining problems, either the negative or positive stimulus of one discrimination was overtrained. In the former condition, on test trials, the LT negative stimuli were preferred, while in the latter, the MT negative stimuli were preferred. These results could be accounted for by the relative frequency during training of nonrewarded responses to the MT and LT negative stimuli.
\end{abstract}

The nonreinforcement of a response to the negative stimulus in a two-choice simultaneous discrimination appears, in naive animals, not to be aversive, at least in the sense that the probability of response to the stimulus has not been reduced (Behar, 1961). In the study noted, naive monkeys responded about equally as often to a stimulus that was associated with no reward on half of the trials and a double reward on the remaining trials as they did to a stimulus that was associated on all trials with a single reward. Discrimination learning-set sophisticated monkeys, on the other hand, strongly avoided the stimulus associated with partial nonreward.

*This experiment developed out of a discussion with Stanton Wharburton III; animals were tested by Daniel Fox. Both contributions are gratefully acknowledged. Address requests for reprints to Isaac Behar, USAARL, P.O. Box 577, Fort Rucker, Alabama 36360 .
However, even in sophisticated monkeys, avoidance of the negative stimulus is absent unless the animal has actually responded to it (Behar, 1962; Leary, 1956). The negative stimulus which has not been responded to, when paired with a neutral stimulus, is preferred at levels well above chance expectation. The negative stimulus which has been responded to is avoided, but to a decreasing degree, as the number of intervening error-free trials increases (Behar, 1962).

This waning in avoidance of the negative stimulus with continued training has also been seen by Deutsch \& Biederman (1965) in a paradigm in which the negative stimuli of a relatively more trained (MT) and less trained (LT) discrimination were paired for choice. In that study, 10 of 12 rats at one level of training and 7 of 12 rats at another chose the MT negative stimulus. Since the 